\title{
Cardiorenal Syndrome in Western Countries: Epidemiology, Diagnosis and Management Approaches
}

\author{
Claudio Ronco ${ }^{a}$ Luca Di Lullob

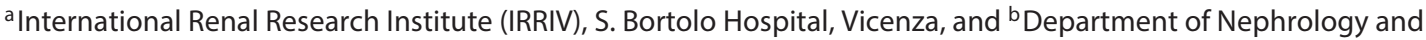 \\ Dialysis, L. Parodi-Delfino Hospital, Colleferro, Italy
}

\section{Key Words \\ Cardiorenal syndrome - Western countries - Risk factors . \\ Diagnosis · Outcomes · Treatment}

\begin{abstract}
Background: It is well established that a large number of hospitalized patients present various degrees of heart and kidney dysfunction; primary disease of the heart or kidney often involves dysfunction or injury to the other. Summary: Based on above-cited organ cross-talk, the term cardiorenal syndrome (CRS) was proposed. Although CRS was usually referred to as abruption of kidney function following heart injury, it is now clearly established that it can describe negative effects of an impaired renal function on the heart and circulation. The historical lack of clear syndrome definition and complexity of diseases contributed to a waste of precious time especially concerning diagnosis and therapeutic strategies. The effective classification of CRS proposed in a Consensus Conference by the Acute Dialysis Quality Group essentially divides CRS into two main groups, cardiorenal and renocardiac CRS, on the basis of primum movens of disease (cardiac or renal); both cardiorenal and renocardiac CRS are then divided into acute and chronic according to disease onset. Type 5 CRS integrates all cardiorenal involvement in-
\end{abstract}

duced by systemic disease. Key Messages: Prevalence and incidence data show a widespread increase of CRS also due to an increasing incidence of acute and chronic cardiovascular disease, such as acute decompensated heart failure, arterial hypertension and valvular heart disease. Patients with chronic kidney disease present various degrees of cardiovascular involvement especially due to chronic inflammatory status, volume and pressure overload and secondary hyperparathyroidism leading to a higher incidence of calcific heart disease. The following review will focus on the main aspects (epidemiology, risk factors, diagnostic tools and protocols, therapeutic approaches) of CRS in Western countries (Europe and United States).

(c) 2016 S. Karger AG, Basel

\section{Background}

The term cardiorenal syndrome (CRS) has been used to define different clinical conditions in which heart dysfunction and kidney dysfunction overlap; its complexity

For advances in the management of acute cardiorenal syndrome in China, see Hou and Yang, Kidney Dis 2016;2:145-150.

\section{KARGER}

E-Mail karger@karger.com www.karger.com/kdd
(C) 2016 S. Karger AG, Basel

2296-9381/16/0024-0151\$39.50/0
Luca Di Lullo, MD, PhD

Department of Nephrology and Dialysis

L. Parodi-Delfino Hospital, Piazza Aldo Moro 1

IT-00034 Colleferro (Roma) (Italy)

E-Mail dilulloluca69@gmail.com 
Table 1. Classification of CRS

\begin{tabular}{|c|c|c|c|}
\hline Type & Denomination & Description & Example \\
\hline 1 & Acute cardiorenal & Heart failure leading to AKI & $\begin{array}{l}\text { Acute coronary syndrome leading to acute heart } \\
\text { and kidney failure }\end{array}$ \\
\hline 3 & Acute nephrocardiac & AKI leading to acute heart failure & Uremic cardiomyopathy AKI related \\
\hline 4 & Chronic nephrocardiac & CKD leading to heart failure & $\begin{array}{l}\text { Left ventricular hypertrophy and diastolic heart } \\
\text { failure due to kidney failure }\end{array}$ \\
\hline
\end{tabular}

needs to be explained beginning from its epidemiological data and risk factor analysis to diagnostic, clinical and therapeutic pathways [1]. An effective classification of CRS was proposed at a Consensus Conference by the Acute Dialysis Quality Group [2] in 2008 (table 1).

\section{CRS Type 1 (Acute CRS)}

\section{Definition and Epidemiological Data}

Type 1 CRS occurs in about $25 \%$ of patients hospitalized for acute decompensated heart failure (ADHF) [3]; among these patients, a preexistent chronic kidney disease (CKD) is common and contributes to acute kidney injury (AKI) in $60 \%$ of all cases studied. AKI can be considered an independent mortality risk factor in ADHF patients, including those with ST myocardial infarction and/or reduced left ventricular ejection fraction (EF) [3].

\section{Risk Factors}

Type 1 CRS (acute cardiorenal) is characterized by the acute worsening of cardiac function leading to AKI [2]. CRS 1 usually presents in the setting of an acute cardiac disease such as ADHF, and it can follow an ischemic (acute coronary syndrome, cardiac surgery complications) or nonischemic (valvular disease, pulmonary embolism) heart disease (fig. 1).

Hemodynamic mechanisms probably play a major role in CRS type 1 in the presence of an ADHF, leading to a decreased renal arterial flow and a consequent fall in GFR. Nonhemodynamic mechanisms have also been proposed to be involved in type $1 \mathrm{CRS}$, including the sympathetic nervous system and renin-angiotensin aldosterone system (RAAS) activation, chronic inflammatory status and impairment of ROS/NO production. Finally, iatrogenesis should also be considered in the pathophysi- ology of type 1 CRS; pharmacological treatment of diabetes mellitus (metformin can provide a negative inotropic effect because of lactic acid production), oncologic diseases (chemotherapeutic agents lead to uric acid abnormal production with direct inhibitory effects on the myocardium and the tubulointerstitial component of the kidney), drug abuse (antibiotics, combined therapy with angiotensin-converting enzyme (ACE) inhibitors and angiotensin II receptor blockers, nonsteroidal anti-inflammatory agents), and heart failure itself can contribute to the onset or worsening of type 1 CRS [4-6].

\section{Diagnosis}

Many biomarkers have been proposed for early diagnosis of kidney injury in type 1 CRS. Cystatin C represents a valid surrogate to test renal function, and it has been recognized as more predictive of long-term mortality and rehospitalization for ADHF than serum creatinine or serum BNP [7]. Other tubular injury biomarkers are represented by kidney injury molecule 1 (KIM-1), liver type fatty acid-binding protein (L-FABP) and interleukin-18 (IL-18), while neutrophil gelatinase-associated lipocalin (NGAL) correlates with renal function markers [8], adverse cardiovascular outcomes or death [8] in ADHF patients. Type 1 CRS can also be diagnosed by bioelectrical devices. The latter allow fluid assessment, which is important in these patients because an association between decreased impedance values (increased body fluid volume) and adverse events such as rehospitalization and death has been demonstrated [8].

Echocardiography shows abnormal myocardial kinetics (indicating an ischemic condition) and left ventricular hypertrophy, valvular stenosis and/or regurgitation, pericardial effusions, normal inspiratory collapse of the inferior vena cava (excluding severe hypervolemia), aortic aneurysms or dissection [9]. The ultrasound of the kid- 
Fig. 1. Pathophysiology of type 1 CRS.

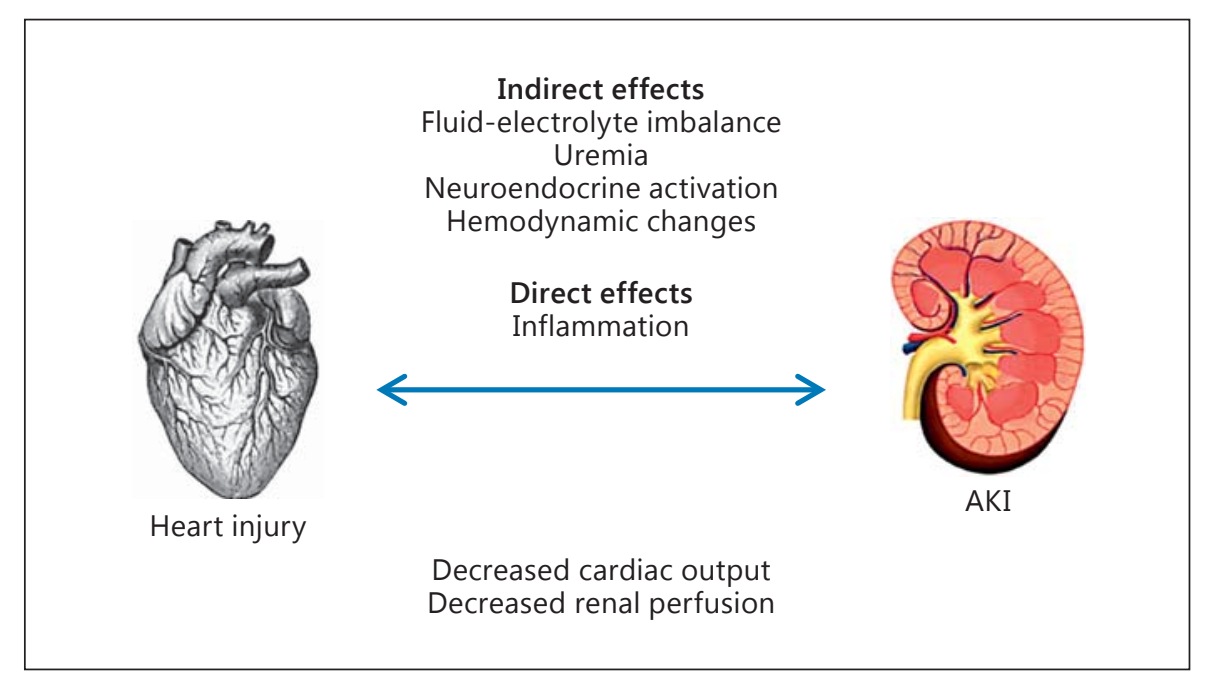

neys usually shows normal or larger dimensions with a preserved cortical-medullary ratio, while color Doppler evaluation shows regular intraparenchymal blood flow, often associated with a raised resistance index $(>0.8 \mathrm{~cm} / \mathrm{s})$ [9].

\section{Management}

Diuretics, beta-blockers, ACE inhibitors or angiotensin receptor blockers, should be started or maximized in patients in the setting of ADHF. The role of B-type natriuretic peptide (nesiritide) in ADHF management remains controversial. Nesiritide may have a role in the management of moderate to severe heart failure in the absence of hypotension. Management of patients with cardiogenic shock can be challenging because of the limited effectiveness of pharmacological therapy. Among patients requiring emergency CABG surgery, the in-hospital mortality rate in Europe and the USA is about 20\%, with a high incidence of stroke (8\%), renal failure requiring dialysis (8.3\%) and bleeding (63.3\%) [10]. Inotropic support remains the central therapy for a depressed myocardium, and correction of the underlying cause such as ischemia will improve outcomes and produce less kidney injury.

\section{CRS Type 2 (Chronic CRS)}

\section{Definition and Epidemiological Data}

CRS type 2 is characterized by chronic abnormalities in cardiac function leading to kidney injury or dysfunction (fig. 2); the temporal relationship between heart and kidney disease is an epidemiological and pathophysiological aspect of the definition itself. CKD has been observed in $45-63 \%$ of chronic heart failure (CHF) patients [11], but it is unclear how to classify these patients, which often include those shifting from a clinical condition of type 1 CRS [12].

\section{Diagnosis}

Assessment of kidney injury in chronic heart failure patients has been previously limited to creatinine (and eGFR) and urinary protein excretion assay. Cardiovascular death and hospitalization in patients with preserved or reduced left ventricular EF as well as eGFR and albuminuria levels are prognostic for renal outcomes in CKD patients [13] but not in heart failure patients [13]. Recently, novel kidney biomarkers (cystatin C, NGAL, KIM-1 and $\mathrm{N}$-acetyl- $\beta$-D-glucosaminidase - NAG) have been evaluated in CHF patients [14], and their plasmatic levels could have prognostic properties in terms of cardiovascular but not renal outcomes.

Kidney ultrasound shows the reduction of cortical thickness, corticomedullary ratio and increased parenchymal echogenicity. Echocardiography shows high atrial volumes or areas as indices of volume overload, normal or decreased EF, right chamber dilation and increased pulmonary arterial pressure, pericardial effusion, and valvular disease (calcific disease) [9].

\section{Management}

In CRS type 2 patients, the main issues concern preventing new-onset renal dysfunction, emerging in a setting of $\mathrm{CHF}$, and counteracting renal dysfunction once it 


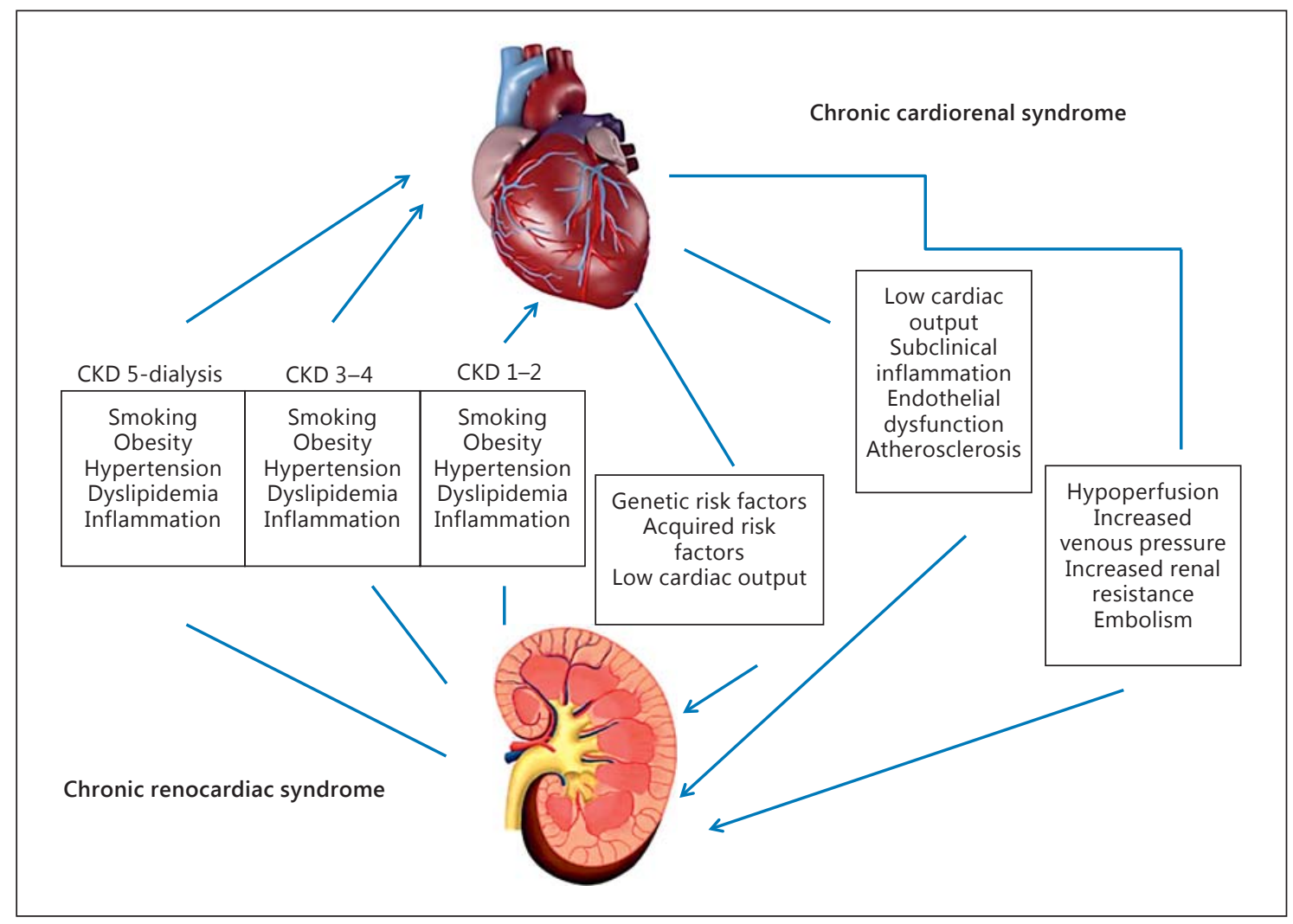

Fig. 2. Pathophysiology of type 2 CRS.

has developed. In this context, attention has to be paid to several drugs used in the management of CHF that may worsen renal function. Diuresis-associated hypovolemia, early introduction of the RAAS blockade and drug-induced hypotension have all been suggested to contribute to the genesis or aggravation of CRS type 2 [15]. In addition, the concept of resistance to diuretics is controversial: diuretic resistance can be suspected when the urine output is relatively poor (for example, $<1,000 \mathrm{ml}$ per day), in spite of the maximal tolerated oral dose of a loop diuretic (for example, $250 \mathrm{mg}$ of furosemide per day), and in the presence of signs and symptoms of refractory hydrosaline retention. The physician has to shift therapy by combining thiazide diuretics with loop diuretics (to block increased distal sodium reabsorption) or by adopting the IV method of administration for loop diuretics (to be given at the same doses or at higher doses compared to those given orally) or, finally, using continuous diuretic infusions to avoid the phenomenon of postdiuretic salt retention; aldosterone receptor antagonists should be taken into consideration as an adjunctive treatment to resolve congestion and reduce the diuretic dose $[16,17]$. Use of high doses of IV loop diuretics in patients with CHF, in whom signs and symptoms are adequately controlled, should be strongly discouraged because of the abovementioned side effects (hypokalemia, hypotension, marked neurohormonal activation and possible renal impairment). Iatrogenic influences may often account for renal damage as much as the congestive nephropathy itself $[18,19]$.

\section{Ultrafiltration for CRS Type 2}

The rationale for isolated ultrafiltration (IUF) in the setting of CRS type 2 is rapid correction of fluid overload when standard management (for example, high-dose IV diuretics with or without inotrope agents) has failed [18, 19]. The current American Heart Association/American College of Cardiology and the European Society of Cardiology treatment guidelines state that IUF is a reasonable option in patients with congestion when the altered fluid status in decompensated patients has been shown not to respond satisfactorily to medical therapy (class IIa, level 
Fig. 3. Pathophysiology of type 3 CRS.

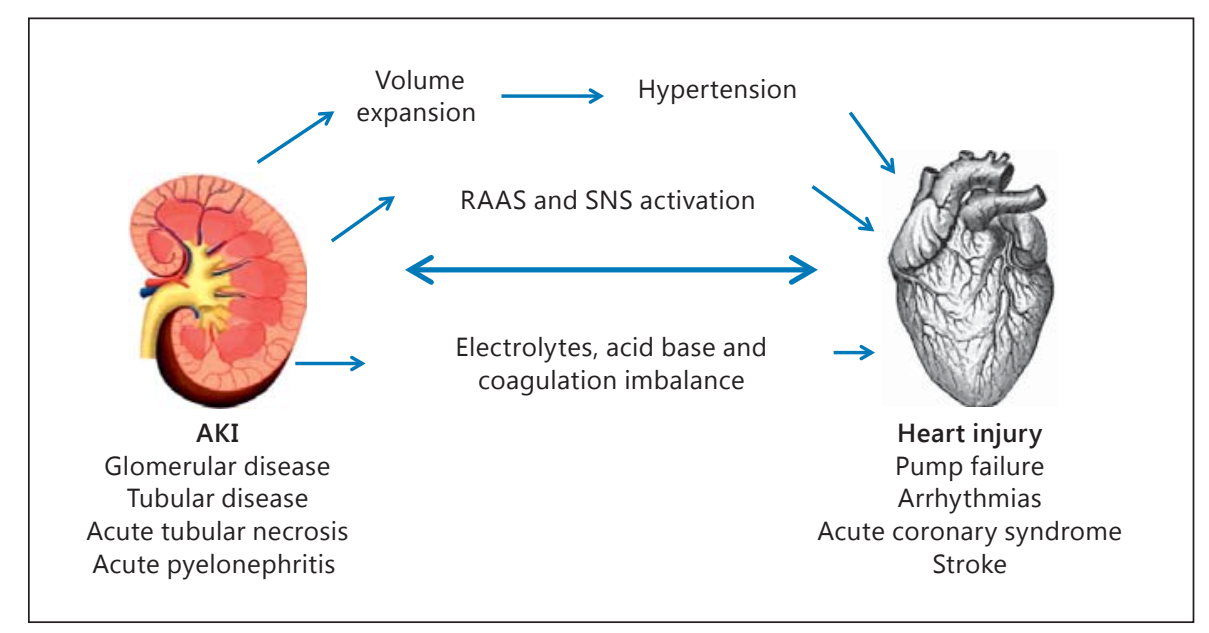

of evidence B) [20]. IUF leaves the plasma concentration of low-molecular-weight solutes, such as sodium and other small solutes, unchanged. IUF does not affect blood electrolytes and urea levels and does not include partial or total replacement by a clean solution with known electrolyte concentrations, as in the case of hemofiltration that holds a more effective depurative efficiency [21].

\section{CRS Type 3 (Acute Renocardiac Syndrome)}

\section{Definition, Epidemiology and Risk Factors}

Type 3 CRS is characterized by acute worsening of kidney function leading to heart disease. A wide spectrum of cardiac dysfunction includes ADHF, acute coronary syndrome and arrhythmias as defined by the RIFLE (Risk, Injury, Failure, Loss, End-stage kidney disease) and AKIN (Acute Kidney Injury Network) criteria [22, 23]. AKI actually represents an independent cardiovascular risk factor for mortality in hospitalized patients especially in those on renal replacement therapy (RRT).

AKI is particularly predominant in patients aged over 65 years with infections at admission, underlying cardiovascular disease, hepatic cirrhosis, respiratory distress, chronic heart failure and hematologic neoplasia. AKI is prevalent in intensive care units (ICUs), mainly due to sepsis, major surgery proceedings, hypovolemic status with low cardiac output heart failure and drug management [24]. AKI seems to involve almost $70 \%$ of patients in ICUs, where $5-25 \%$ of patients can develop severe AKI with mortality rates ranging from 50 to $80 \%$ [3]. ADHF still represents the most common acute cardiac dysfunc- tion syndrome worldwide, and it can be defined as newonset or gradual or rapid worsening of preexistent heart failure with signs and symptoms requiring immediate therapy [25]. Cardiac valvular disease, atrial fibrillation, arterial hypertension as well as noncardiac comorbidities (renal dysfunction, diabetes, anemia) and medications (especially nonsteroidal anti-inflammatory drugs and glitazones) can contribute to ADHF development [25]. Renal dysfunction affects mortality rates in ADHF patients from 1.9 (mild renal disease) to $7.6 \%$ (severe renal dysfunction) according to the Acute Decompensated Heart Failure National Registry database [26]. Adverse prognostic factors are mainly represented by low EF, low systolic blood pressure, hyponatremia, older age and inflammatory status with elevated C-reactive protein (CRP) plasmatic levels [27]. Probably, AKI and ADHF could be identified as two sides of the same coin, and a vicious cycle is established: AKI leads to heart dysfunction, and heart disease affects progressive kidney failure, and many comorbidities could lead to AKI (fig. 3).

An AKI incidence of 2,147 per million population was reported in a population-based study from northern Scotland [28]. Another prospective, multicenter, communitybased study in 748 AKI patients reported common causes of death: infections (48\%), hypovolemic shock (45.9\%), respiratory distress $(22.2 \%)$, heart disease (15\%), disseminated intravascular coagulation $(6.3 \%)$, gastrointestinal bleeding (4.5\%) and stroke (2.7\%). In a more recent retrospective AKI study following traumatic disease, cardiac arrest was reported as a cause of death in $20 \%$ of patients. Other causes of death were cerebrovascular accidents $(46 \%)$, sepsis (17\%), multiple organ dysfunction syndrome (7.3\%) and respiratory insufficiency (3.2\%) [29]. 


\section{Diagnosis}

Ultrasound Diagnosis

Kidney size and echogenicity provide primary features to discern between acute and chronic nephropathies, but it must be remembered that chronic renal failure does not exclude normal or enlarged kidneys (e.g. early stages of diabetic nephropathy, HIV-related glomerulonephritis or cast nephropathy). A hyperechogenic renal cortex with low corticomedullary ratio is predictive of chronic nephropathy [9], insofar as intraparenchymal resistance index increases [9]. An echocardiographic pattern is not diagnostic, showing an increase in atrial volumes or areas as indices of volume overload, pleural or pericardial effusion, and it is often associated with the evidence of lung comets on thoracic ultrasound [9].

Biochemical Diagnosis (Biomarkers) (table 2)

AKI Biomarkers. NGAL is a protein of the lipocalin superfamily, and it is normally expressed (at low levels) by neutrophils and several epithelial cells (kidney, lung, stomach and gut). NGAL seems to play an important role in limiting oxidative damage in acute and chronic kidney diseases, and it represents the earliest kidney biomarker of ischemic damage. KIM-1 is a transmembrane glycoprotein, normally undetected in the urinary samples, that can be found in the urine after ischemic or nephrotoxic insult to proximal tubular cells; KIM-1 urinary levels seem to be highly specific for ischemic AKI (such as acute tubular necrosis) [7]. L-FABP is a protein mainly produced in the liver and expressed on hepatocytes and renal proximal tubular cells; it can be filtered by renal glomeruli and reabsorbed in the proximal tubular cells; if renal proximal tubular cells are injured, L-FABP urinary levels rapidly increase [7] but later in respect of NGAL. IL-18 is a proinflammatory cytokine detected in the urine after ischemic tubular damage and associated with AKI mortality and sepsis. Netrin-1 is a laminin-like protein whose blood levels can increase in AKI patients; it can be detected in urine 1-3 h after ischemia-reperfusion following renal injury [7].

Cardiac Biomarkers. BNP is a vasopeptide hormone released by the left ventricle in response to wall stress and modified by a prohormone (proBNP). The BNP/NT proBNP ratio is the best diagnostic and prognostic marker in patients with acute renal failure [30].

Troponins are highly sensitive and specific for ischemic myocardial injury, and they correlate with outcomes in kidney disease patients [31]. Heart-FABP (H-FABP) is a nonenzymatic protein increasing during cardiac ischemia, and it holds more than $80 \%$ sensitivity for diagnosis
Table 2. Potential biomarkers of AKI, acute cardiac dysfunction and type 3 CRS

Potential biomarkers for early detection of AKI

NGAL

KIM-1

Cystatin C

IL-18

NAG

L-FABP

Netrin-1

Klotho

Midkine

Potential biomarkers for differential diagnosis of AKI

KIM-1

IL-18

Potential for prognosis of AKI

NGAL

Cystatin C

NAG

Potential biomarkers of inflammation and immune response

Urinary IL-18

Tumor necrosis factor receptor-1

Urinary vascular cell adhesion molecules-1

Monocyte chemoattractant protein-1

Early detection of acute cardiac dysfunction

$\mathrm{BNP} / \mathrm{NT}$-proBNP

cTnT, cTnI

Myoglobin

Myeloperoxidase

CRP

H-FABP

of acute myocardial infarction in the period of 30-210 min after symptom onset [31], faster than CK-MB activity and cardiac troponins, but it shows limited diagnostic value in kidney disease patients [31].

\section{Management}

To better provide complete management of type-3 CRS, the best treatment strategy is probably to identify various stages of disease (according to the RIFLE/AKIN criteria).

\section{Patients at High Risk of Developing AKI}

Avoiding or minimizing nephrotoxic medications and procedures is an important strategy to prevent AKI. Antibiotics (aminoglycosides) and contrast medium represent the main nephrotoxic agents employed in ICUs, and their employment should be reduced. Combination therapy with vancomycin and aminoglycoside or ACE inhib- 
itors, nonsteroidal anti-inflammatory drugs and diuretics can lead to renal tubular injury and volume depletion. Preventing hypoperfusion is a cornerstone to avoid AKI, and volume depletion should be corrected. Strict monitoring of fluid balance is fundamental to avoid volume overload, especially in patients with higher filling pressures and signs of right heart dysfunction due to increased preload.

\section{Stage 1 (Risk)}

Patients at risk fulfill AKI criteria, and they can develop severe AKI and acute renal failure. These patients should be treated as the previous group; in addition, they need urine analysis, routine blood tests, biomarkers and ultrasound to investigate the etiology, make diagnosis, and plan the treatment. Close monitoring and supportive care should be provided [32].

\section{Stage 2 (Injury)}

Stage 2 patients are characterized by a high risk of morbidity/mortality due to renal injury. Patients need conservative therapy and functional hemodynamic monitoring to guide resuscitation, especially pulse pressure variation in ventilated patients should be considered. Maintenance of electrolytes and acid-base homeostasis should be ensured. Drug dosing and their blood levels have to be in the therapeutic range to avoid incorrect storage [32].

\section{Stage 3 (Failure)}

At this stage, the patient is at the highest risk of death and has a high probability of extrarenal complications including CRS. RRT or extracorporeal kidney support should be considered if pharmacological therapy does not work, kidney injury is severe, or there is a risk of developing life-threatening complications. Prevention of left ventricular volume overload is critical to maintain adequate cardiac output and systemic perfusion. Continuous infusion of furosemide has been recommended for improved efficacy and combination therapy with thiazide diuretics [32].

\section{Renal Replacement Therapy}

Once pharmacological treatment has failed in AKI patients and oligo-anuric renal failure has been established, RRT has to be started. The timing of RRT initiation is strongly dependent on clear impairment of renal function with electrolyte and acid-base imbalance, hypercreatininemia and severe fluid overload not responsive to pharmacological treatment [33]. RRT initiation can impact on clinical outcomes; for example, early application of RRT

Cardiorenal Syndrome in Western

Countries in patients with severe sepsis might be beneficial but, at the same time, an early 'classic dose' of continuous venovenous hemofiltration does not provide complete or partial renal recovery [33].

It has also been demonstrated that avoiding or delaying RRT is strictly associated with higher mortality and increased hospitalization rates [34]. RRT can be stopped when improvement in renal function is clearly evident as pointed out by increased urine output and a decrease in serum creatinine levels in patients with a continuous RRT (CRRT) dose. A urine output of more than $400 \mathrm{ml} /$ day can represent a cutoff value, while a $15-20 \mathrm{ml} / \mathrm{min}$ creatinine clearance could allow CRRT withdrawal [35].

CRRT and intermittent hemodialysis (IHD) both present pros and cons; when correctly applied, both CRRT and IHD can achieve good metabolic control in many randomized controlled trials and meta-analyses [35], although CRRT seems to be associated with best outcomes and more frequent renal recovery in critically ill patients in comparison with IHD [35].

Together with CRRT and IHD therapies, some 'hybrid therapies' have been proposed, such as sustained low-efficiency (daily) dialysis (SLED) and extended daily dialysis in which IHD techniques are adapted to provide longer dialysis sessions [36]. Some clinical trials have not found any difference between SLED and continuous venovenous hemofiltration in terms of cardiovascular stability and mortality rates, but SLED seems to be associated with a short duration of mechanical ventilation [36]. Concerning RRT, it was largely underdosed in critically ill patients in the past decades; the Vicenza group trial proposed the milestone RRT dose of $35 \mathrm{ml} / \mathrm{kg}$ to be increased in septic patients [37], while large multicenter randomized controlled trials have proposed a $20-30 \mathrm{ml} /$ $\mathrm{kg} / \mathrm{h}$ dose for CRRT treatments [37]. In summary, clinicians have to tailor RRT therapy to individual critically ill patients to provide the ideal blood purification treatment.

\section{CRS Type 4 (Chronic CRS)}

\section{Definition and Epidemiology}

Type 4 CRS, also defined as chronic renocardiac disease, is characterized by cardiovascular involvement in patients affected by CKD at any stage. It is well established that renal dysfunction is an independent risk factor for cardiovascular disease with a higher mortality risk for myocardial infection and sudden death in CKD. A metaanalysis by Tonelli et al. [38] conducted on 1.4 million patients found higher mortality rates for all causes with 


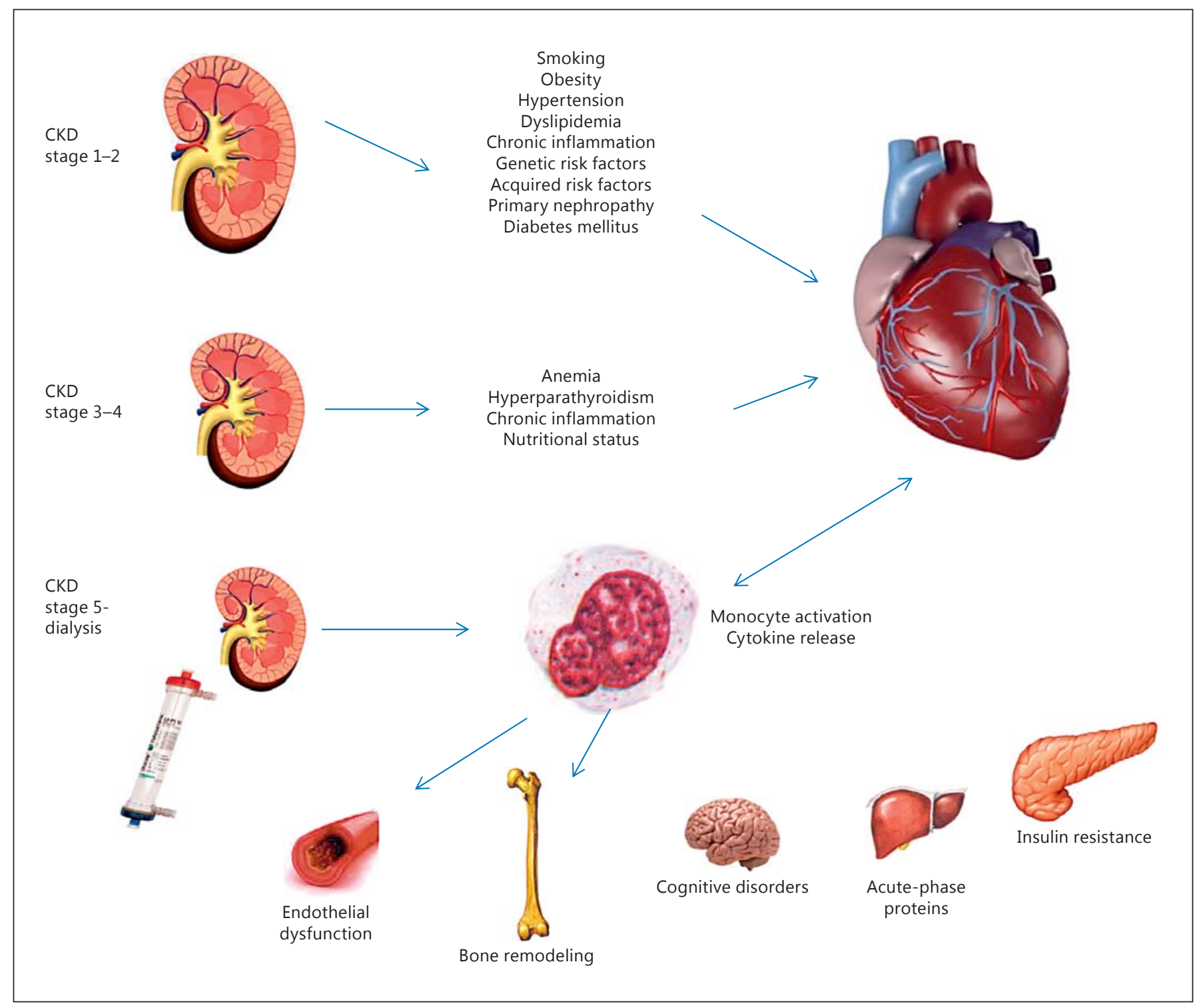

Fig. 4. Pathophysiology of type 4 CRS.

eGFR decline with relative death odds ratios of 1.9, 2.6 and 4.4 for GFR levels of 80,60 and $40 \mathrm{ml} / \mathrm{min}$, respectively.

The largest epidemiological study was actually performed by Go et al. [39] on over 1 million people: cardiovascular risk was found particularly evident in patients with stages IIIb-IV (according to the K/DOQI CKD classification) renal disease and in those who underwent RRT (hemodialysis, peritoneal dialysis and transplantation).

Chronic Renal Insufficiency Cohort (CRIC) Study investigators focused their attention on 190 patients pre- senting stage III to end-stage renal disease and performing serial echocardiographic exams; in the 2-year evaluation period in which patients shifted from stage $\mathrm{V}$ to end-stage renal disease, the EF dropped from 53 to $50 \%$; therefore, they found that the number of subjects with $\mathrm{EF}$ $<50 \%$ increased by $20 \%[40,41]$.

\section{Risk Factors}

Figure 4 shows close interactions between CKD and cardiovascular involvement. CKD can indirectly (exacerbating ischemic heart disease) and directly (pressure and 
volume overload leading to left ventricular hypertrophy) contribute to heart disease [41]. Left ventricular hypertrophy is highly prevalent in patients starting hemodialysis, and it is accountable for subsequent hospitalizations for heart failure [41]; pressure overload leading to left ventricular hypertrophy results from comorbid conditions such as hypertension and calcific valvular disease particularly prevalent in hemodialysis and predialysis patients [41]. Hyperphosphatemia and secondary hyperparathyroidism (also described as CKD mineral and bone disorder) can produce the ossification of cardiac vessels and valves because of the 'osteoblastic' transformation of vascular smooth muscle cells [41]. Hypertension itself can also contribute to vascular calcification determining pressure overload. CKD patients, especially those undergoing dialysis treatment, are more prone to develop arrhythmias, especially atrial fibrillation and ventricular tachyarrhythmias.

Almost half of cardiovascular deaths in the end-stage kidney disease population are related to cardiac arrhythmia or sudden death [42]. The increased risk for sudden death seems to be particularly related to longer dialytic intervals in subjects undergoing thrice weekly hemodialysis treatment because of extreme shifts of electrolytes and fluids [42].

While most of the clinical studies have been focused on sudden death, several investigators have given more attention to prevalence and incidence of atrial fibrillation in CKD and ESRD patients; in the Chronic Renal Insufficiency Cohort (CRIC) study, an 18\% prevalence of atrial fibrillation was found [41]. Volume overload is mainly supported by CKD secondary anemia and sodium and water retention, and it can be worsened by the presence of hemodialysis vascular access [41]. Chronic inflammation, insulin resistance, hyperhomocysteinemia and lipid dysmetabolism can also contribute to cardiovascular disease in CKD patients [43].

\section{Diagnosis}

Cardiac function is more widely assessed by NT-proBNP serum levels, while eGFR represents the main biochemical test to evaluate kidney function. Kidney ultrasound exam shows features of chronic nephropathy such as a thin and hyperechogenic cortex with a reduced cortico medullary ratio together with a small dilation of the urinary tract; parapelvic and subcortical cysts are also found [9]. Echocardiography underlines signs of volume overload, left ventricular dysfunction and right ventricular dysfunction especially in ESRD and hemodialysis patients.
Increased atrial volumes or areas, pleural or pericardial effusion and lung comets are suggestive of volume overload [9]. It is quite common to describe valvular calcifications (related to secondary hyperparathyroidism) [9] and frequent right heart dysfunction features such as high pulmonary artery pressure, low tricuspid annulus plane systolic excursion (TAPSE) or right chamber dilation [44].

\section{Management}

Looking at the RAAS system activation in CKD patients, the RENAAL study was one of the cornerstones in this field of application. RENAAL investigators aimed to evaluate renoprotective effects of losartan in over 1,500 type 2 diabetic patients with renal involvement without evidence of heart failure at baseline [45]. Quite similar to the RENAAL study, the Irbesartan Diabetic Nephropathy Trial (IDNT) study was designed to evaluate renoprotective effects of irbesartan versus amlodipine or placebo in over 1,700 patients [46]. The results showed that the irbesartan group had a lower incidence of heart failure compared to the amlodipine and placebo groups. The VAL-HeFT (Valsaran in Heart Failure Trial) study showed higher mortality and morbidity rates in patients with a GFR $<60 \mathrm{ml} / \mathrm{min} / 1.73 \mathrm{~m}^{2}$ and proteinuria, lower rates in those with no proteinuria and normal GFR, and intermediate rates in the remaining patients; therefore, the CKD patients randomized to valsartan therapy had lower rates of first heart failure hospitalization or death from cardiovascular disease [47]. The use of beta-blockers together with ACE inhibitors or angiotensin II receptor blockers is associated with better cardiovascular and renal outcomes in elderly patients, and also those with advanced CKD [48]. In the Evaluation of Cinacalcet Hydrochloride Therapy to Lower Cardiovascular Events (EVOLVE), a reduction in the first heart failure episode was reported in the cinacalcet group [49]. Di Lullo et al. [50] found that treating predialysis patients with sevelamer hydrochloride $(1,600$ $\mathrm{mg} /$ day), a calcium-free phosphate binder, both a reduction of cardiac valve calcifications and delay in kidney function decline occurred. Dyslipidemia represents another fundamental target to achieve in managing cardiovascular complications in CKD patients; the SHARP trial actually represents the largest trial on statin employment in CKD patients showing a significant benefit of the combination simvastatin/ezetimibe on major atherosclerotic events, although all-cause mortality was unaffected [51]. 


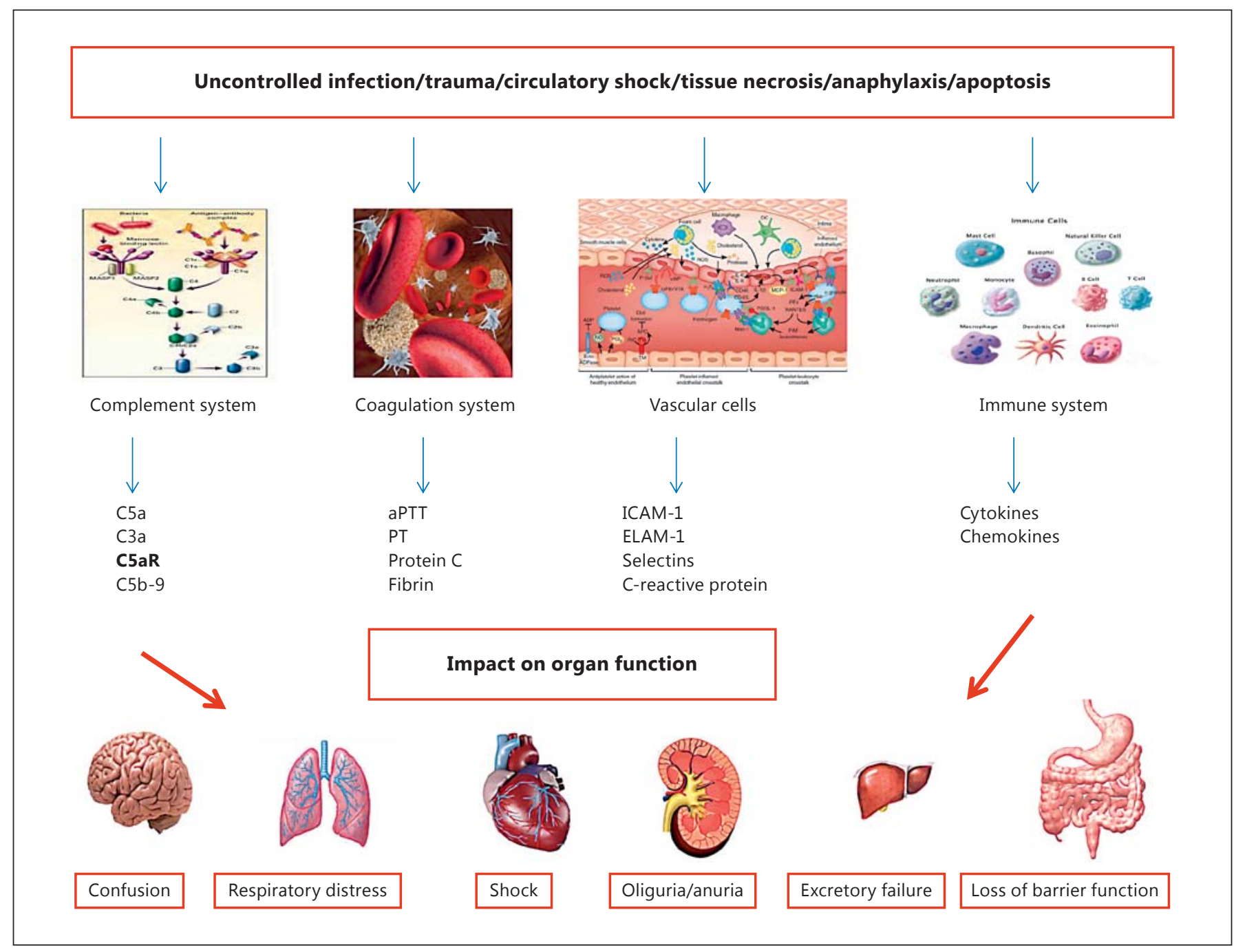

Fig. 5. Pathophysiology of type 5 CRS. aPTT = Activated partial thromboplastin time; PT = prothrombin time.

\section{CRS Type 5 (Secondary CRS)}

\section{Definition and Epidemiology}

Type 5 CRS is a recently defined clinical syndrome, and complete epidemiological data on this entity are still incomplete. Type 5 CRS occurs when cardiac and renal injury occur simultaneously as it occurs in sepsis, where heart and kidney are involved secondary to a common underlying pathological trigger [2].

\section{Risk Factors}

Acute CRS- 5 results from systemic processes, for example sepsis, infections, drugs, toxins and connective tissue disorders such as lupus, granulomatosis with polyan- giitis (Wegener's) and sarcoidosis (fig. 5). On the other hand, in cirrhotic liver disease patients, CRS- 5 has a more insidious onset, and the kidney and cardiac dysfunction may develop slowly until a crucial point is reached and full decompensation occurs. Several factors influence the course of CRS-5. Chronic CRS-5 (i.e. CRS in cirrhotic patients) presents a quite variable time sequence because in most cases of CRS- 5 there is an underlying condition and related precipitating event that signals its presence. For instance, cirrhotic patients are subject to infections and an acute CRS-5 can overlap a chronic process [43].

The pathophysiological changes in sepsis-related CRS can depend on the systemic effects of sepsis itself. Concerning sepsis-related kidney involvement, there are clear 
alterations in intraparenchymal blood flow independent of systemic hemodynamic changes linked to septic process [43]. Sepsis, therefore, is able to affect the autonomic nervous system (ANS), RAAS and hypothalamus-pituitary gland-adrenal gland axis which can affect, in several and distinctive steps, cardiac and/or renal function. The severity of ANS dysfunction correlates with morbidity and mortality [52]; autonomic dysfunction can be assessed by observing decreased heart rate variability, often associated with the release of inflammatory biomarkers such as IL-6, IL-10 and CRP.

Abnormalities in oxidative stress are also found, from mitochondrial dysfunction to alteration in antioxidant stress enzymes [53]. Contractile heart function is mainly affected, and muscle protein expression (actin and myo$\sin$ ) is abnormal. Sepsis induces tubular damage in kidneys affected by increased secretion of lipopolysaccharide that alters $\mathrm{HCO}_{3}$ transport leading to abnormalities in urine acidification [53].

\section{Diagnosis}

A recent review has pointed out some characteristic biomarkers whose elevation is typical during the septic process: lipopolysaccharide-binding protein, procalcitonin, CRP, proinflammatory cytokines (IL-6, TGF- $\beta$ ) [54]. Assessment of cardiac function in type 5 CRS is quite similar to other clinical situations in which myocardial dysfunction is present. Natriuretic peptides and troponin level assays provide information about cardiac chambers (especially left cardiac chambers) and myocardial cell damage. In the early stages of the septic process, there is a low-output myocardial involvement; after starting fluid therapy, the clinical picture shifts to a typical distributive shock characterized by an increased cardiac output and systemic vasodilatation [43]. The echocardiographic assay confirms high-output cardiomyopathy with abnormalities in the left ventricular regional contractility together with the dilation of left heart chambers [9]. The diagnosis of kidney involvement in sepsis-related type 5 CRS overlaps other forms of AKI with acute changes in serum creatinine levels according to the RIFLE, AKIN and KDIGO criteria [43].

\section{Management}

First of all, maintaining hemodynamic stability and guaranteeing tissue perfusion are the key points to prevent type $5 \mathrm{CRS}$ in the hyperacute phase of sepsis together with fluid control and correct antibiotic treatment. Fluid therapy must be carefully managed to avoid fluid overload and other iatrogenic complications [43].
Since inflammation and immune disorders play an important role in the pathogenesis of sepsis, removal of cytokines and immunomodulation can be obtained with high permeability membranes [55]. To manage heart complications, an approach with fluid therapy together with vasopressors, vasodilators and inotropes is required for maintaining filling pressures; vasopressors should be carefully administered because of depressive effects on cardiac output. More recently, levosimendan has been proven to provide benefits in decompensated heart failure to increase EF and diuresis; levosimendan efficacy is still to be proven in the prevention of type 5 CRS [43]. Renal support includes the removal of any nephrotoxic drug and media, maintenance of adequate perfusion pressure and, if indicated, early intervention with dialysis therapy [43]. There is no role for dopamine in improving renal hemodynamics [56], and the studies with fenoldopam are limited [57]. Norepinephrine decreases renal perfusion in normal conditions but increases systemic blood pressure in septic patients [56], while vasopressin increases diuresis and GFR in septic patients [56].

Diuretics have a limited role in managing heart and kidney involvement in septic patients [58] and RRT with CRRT should be promptly started [59]; early ultrafiltration seems to improve renal outcomes in septic shock patients, but these data have to be confirmed in further clinical trials.

\section{Conflict of Interest Statement}

The authors declare no conflicts of interest.

References

1 Ronco C: Cardiorenal and renocardiac syndromes: clinical disorders in search of a systematic definition. Int J Artif Organs 2008;31: $1-2$

2 Bargshaw SM, Cruz DM, Aspromonte N, et al; the Acute Dialysis Quality Initiative (ADQI) Consensus Group: Epidemiology of cardio-renal syndromes: workgroup statements from the 7th ADQI Consensus Conference. Nephrol Dial Transplant 2010;25:14061416.

3 Dar O, Cowie MR: Acute heart failure in the intensive care unit: epidemiology. Crit Care Med 2008;36:S3-S8.

4 Van Sloten TT, Pijpers E, Steouwer CD, Brouwers MC: Metformin-associated lactic acidosis in a patient with normal kidney function. Diabetes Res Clinic Pract 2012;96x:57-58. 
5 Prawle JR, Liu YI, Licari E, et al: Oliguria as predictive biomarker of acute kidney injury in critically ill patients. Crit Care 2011;15:R172.

6 Prowle JR, Echeverri JE, Ligabo EV, Ronco C, Bellomo R: Fluid balance and acute kidney injury. Nat Rev Nephrol 2010;6:107-115.

7 Lassus JP, Nieminen MS, Peuhkurinen K, Pulkki K, Siirilä-Waris K, Sund R, Harjola VP; FINN-AKVA Study Group: Markers of renal function and acute kidney injury in acute heart failure: definitions and impact on outcomes of the cardiorenal syndrome. Eur Heart J 2010;31:2791-2798.

8 Cruz DN, Gaiao S, Maisel A, Ronco C, Devarajan P: Neutrophil gelatinase-associated lipocalin as a biomarker of cardiovascular disease: a systematic review. Clin Chem Lab Med 2012;50:1533-1545.

9 Di Lullo L, Floccari F, Granata A, D'Amelio A, Rivera R, Fiorini F, Malaguti M, Timio M: Ultrasonography: Ariadne's thread in the diagnosis of cardiorenal syndrome. Cardiorenal Med 2012;2:11-17.

10 Hollenberg SM, Kevinsky CJ, Parrillo JE: Cardiogenic shock. Ann Intern Med 1999;131: 47-59.

11 Hebert K, Dias A, Delgado MC, Franco E, Tamariz L, Steen D, Trahan P, Major B, Arcement LM: Epidemiology and survival of the five stages of chronic kidney disease in a systolic heart failure population. Eur J Heart Fail 2010;12:861-865.

12 Hillege HL, Nitsch D, Pfeffer MA, Swedberg $\mathrm{K}$, McMurray JJ, Yusuf S, Granger CB, Michelson EL, Ostergren J, Cornel JH, et al: Renal function as a predictor of outcome in a broad spectrum of patients with heart failure. Circulation 2006;113:671-678.

13 Halbesma N, Jansen DF, Heymans MW, Stolk RP, de Jong PE, Gansevoort RT: Development and validation of a general population renal risk score. Clin J Am Soc Nephrol 2011;6: 1731-1738.

14 Cruz DN, Fard A, Clementi A, Ronco C, Maisel A: Role of biomarkers in the diagnosis and management of cardio-renal syndromes. Semin Nephrol 2012;32:79-92.

15 Liang KV, Williams AW, Greene EL, Redfield MM: Acute decompensated heart failure and the cardiorenal syndrome. Crit Care Med 2008;36(suppl):S75-S88.

16 Costanzo MR, Guglin ME, Saltzberg MT, et al; UNLOAD Trial Investigators: Ultrafiltration versus intravenous diuretics for patients hospitalized for acute decompensated heart failure. J Am Coll Cardiol 2007;49:675-683.

17 Shah SU, Anjum S, Littler WA: Use of diuretics in cardiovascular diseases: (1) heart failure. Postgrad Med J 2004;80:201-205.

18 Butler J, Forman DE, Abraham WT, et al: Relationship between heart failure treatment and development of worsening renal function among hospitalized patients. Am Heart J 2004; 147:331-338.

19 Costanzo MR: Ultrafiltration in the management of heart failure. Curr Opin Crit Care 2008; $14: 524-530$
20 Hunt SA, Abraham WT, Chin MH, et al; American College of Cardiology Foundation; American Heart Association: 2009 Focused update incorporated into the ACC/AHA 2005 Guidelines for the Diagnosis and Management of Heart Failure in Adults. A Report of the American College of Cardiology Foundation/American Heart Association Task Force on Practice Guidelines Developed in Collaboration with the International Society for Heart and Lung Transplantation. J Am Coll Cardiol 2009; 53:e1-e90.

21 Fiaccadori E, Regolisti G, Maggiore U, et al: Ultrafiltration in heart failure. Am Heart J 2011;161:439-449.

22 Bellomo R, Ronco C, Kellum JA, Mehta RL, Palevsky PM; the ADQI Workgroup: Acute renal failure - definition, outcome measures, animal models, fluid therapy and information technology needs. The Second International Consensus Conference of the Acute Dialysis Quality Initiative (ADQI) Group. Crit Care 2004;8:R204-R212.

23 Mehta RL, Kellum JA, Shah SV, et al: Acute kidney injury network (AKIN): report of an initiative to improve outcomes in acute kidney injury. Crit Care 2007;11:R31.

24 Uchino S, Kellum JA, Bellomo R, et al: Beginning and Ending Supportive Therapy for the Kidney (BEST Kidney) investigators. Acute renal failure in critically ill patients: a multinational, multicenter study. JAMA 2005;294: 813-818.

25 Gheorghiade M, Zannad F, Sopko G, et al: Failure syndromes: current state and framework for future research. Circulation 2005; 112:3958-3968

26 Sharabas I, Siddiqi N: Cardiovascular disease risk profiles comparison among dialysis patients. Saudi J Kidney Dis Transpl 2016;27: 692-700.

27 Siirila-Waris K, Lassus J, Melin J, et al: Characteristics, outcomes, and predictors of 1 year mortality in patients hospitalized for acute heart failure. Eur Heart J 2006;27:3011-3017.

28 Ali T, Khan I, Simpson W, et al: Incidence and outcomes in acute kidney injury: a comprehensive population-based study. J Am Soc Nephrol 2007;18:1292-1298.

29 De Abreu KLS, Silva Junior GB, Carreto AGC, et al: Acute kidney injury after trauma: prevalence, clinical characteristics and RIFLE classification. Indian J Crit Care Med 2010;14: 121-128.

30 Hillege HL, Girbes AR, de Kam PJ, et al: Renal function, neurohormonal activation and survival in patients with chronic heart failure. Circulation 2000;102:203-210.

31 Di Lullo L, Barbera V, Santoboni A, Bellasi A, Cozzolino M, De Pascalis A, Rivera R, Balducci A, Russo D, Ronco C: Troponins and chronic kidney disease. G Ital Nefrol 2015;32.

32 Chuasuwan A, Kellum JA: Cardio-renal syndrome type 3: epidemiology, pathophysiology, and treatment. Semin Nephrol 2012;32: 31-39.
33 Bellomo R, Kellum JA, Ronco C: Acute kidney injury. Lancet 2012;380:756-766.

34 Karvellas CJ, Farhat MR, Sajjad I, Mogensen SS, Leung AA, Wald R, et al: A comparison of early versus late initiation of renal replacement therapy in critically ill patients with acute kidney injury: a systematic review and meta-analysis. Crit Care 2011;15:R72.

35 Schneider AG, Bellomo R, Bagshaw SM, Glassford NJ, Lo S, Jun M, et al: Choice of renal replacement therapy modality and dialysis dependence after acute kidney injury: a systematic review and meta-analysis. Intensive Care Med 2013;39:987-997.

36 Wu VC, Wang CH, Wang WJ, Lin YF, Hu FC, Chen YW, et al: Sustained low-efficiency dialysis versus continuous veno-venous hemofiltration for postsurgical acute renal failure. Am J Surg 2010;199:466-476.

37 Bellomo R, Cass A, Cole L, Finfer S, Gallagher $\mathrm{M}$, Lo S, et al: Intensity of continuous renalreplacement therapy in critically ill patients. N Engl J Med 2009;361:1627-1638.

38 Tonelli M, Wiebe N, Culleton B, House A, Rabbat C, Fok M, McAlister F, Garg AX: Chronic kidney disease and mortality risk: a systematic review. J Am Soc Nephrol 2006;17: 2034-2047.

39 Go AS, Chertow GM, Fan D, McCulloch CE, Hsu CY: Chronic kidney disease and the risks of death, cardiovascular events, and hospitalization. N Engl J Med 2004;351:1296-1305.

40 Bansal N, Keane M, Delafontaine P, Dries D, Foster E, Gadegbeku CA, Go AS, Hamm LL, Kusek JW, Ojo AO, Rahman M, Tao K, Wright JT, Xie D, Hsu CY; CRIC Study Investigators: A longitudinal study of left ventricular function and structure from CKD to ESRD: the CRIC study. Clin J Am Soc Nephrol 2013;8:355-362.

41 Di Lullo L, House A, Gorini A, Santoboni A, Russo D, Ronco C: Chronic kidney disease and cardiovascular complications. Heart Fail Rev 2015;20:259-272.

42 Di Lullo L, Rivera R, Barbera V, Bellasi A, Cozzolino M, Russo D, De Pascalis A, Banerjee D, Floccari F, Ronco C: Sudden cardiac death and chronic kidney disease: from pathophysiology to treatment strategies. Int J Cardiol 2016;217:16-27.

43 Ronco C, Di Lullo L: Cardiorenal syndrome. Heart Fail Clin 2014;10:251-280.

44 Di Lullo L, Floccari F, Rivera R, Barbera V, Granata A, Otranto G, Mudoni A, Malaguti M, Santoboni A, Ronco C: Pulmonary hypertension and right heart failure in chronic kidney disease: new challenge for 21st-century cardionephrologists. Cardiorenal Med 2013; 3:96-103.

45 Brenner BM, Cooper ME, de Zeeuw D, Keane WF, Mitch WE, Parving HH, Remuzzi G, Snapinn SM, Zhang Z, Shahinfar S; RENAAL Study Investigators: Effects of losartan on renal and cardiovascular outcomes in patients with type 2 diabetes and nephropathy. $\mathrm{N}$ Engl J Med 2001;345:861-869. 
46 Lewis EJ, Hunsicker LG, Clarke WR, Berl T, Pohl MA, Lewis JB, Ritz E, Atkins RC, Rohde R, Raz I; Collaborative Study Group: Renoprotective effect of the angiotensin-receptor antagonist irbesartan in patients with nephropathy due to type 2 diabetes. $\mathrm{N}$ Engl J Med 2001;345:851-860.

47 Lesogor A, Cohn JN, Latini R, Tognoni G, Krum H, Massie B, Zalewski A, Kandra A, Hua TA, Gimpelewicz C: Interaction between baseline and early worsening of renal function and efficacy of renin-angiotensin-aldosterone system blockade in patients with heart failure: insights from the Val-HeFT study. Eur J Heart Fail 2013;15:1236-1244.

48 Cice G, Ferrara L, D’Andrea A, D'Isa S, Di Benedetto A, Cittadini A, Russo PE, Golino P, Calabro R: Carvedilol increases two-year survival in dialysis patients with dilated cardiomyopathy: a prospective, placebo-controlled trial. J Am Coll Cardiol 2003;41:1438-1444.
49 EVOLVE Trial Investigators; Chertow GM, Block GA, Correa-Rotter R, Drueke TB, Floege J, Goodman WG, Herzog CA, Kubo Y, London GM, Mahaffey KW, Mix TC, Moe SM, Trotman ML, Wheeler DC, Parfrey PS: Effect of cinacalcet on cardiovascular disease in patients undergoing dialysis. N Engl J Med 2012;367:2482-2494.

50 Di Lullo L, Floccari F, Santoboni A, Barbera V, Rivera RF, Granata A, Morrone L, Russo D: Progression of cardiac valve calcification and decline of renal function in CKD patients. J Nephrol 2013;26:739-744.

51 Sharp Collaborative Group: Study of Heart and Renal Protection (SHARP): randomized trial to assess the effects of lowering low-density lipoprotein cholesterol among 9,438 patients with chronic kidney disease. Am Heart J 2010;160:785-794.

52 Tateishi Y, et al: Depressed heart rate variability is associated with high IL-6 blood level and decline in the blood pressure in septic patients. Shock 2007;28:549-553.
53 Stengl M, et al: Reduced L-type calcium current in ventricular myocytes from pigs with hyperdynamic septic shock. Crit Care Med 2010;38:579-587.

54 Reinhart K, et al: New approaches to sepsis: molecular diagnostics and biomarkers. Clin Microbiol Rev 2012;25:609-634.

55 Nakamura M, et al: Treatment of severe sepsis and septic shock by CHDF using a PMMA membrane hemofilter as a cytokine modulator. Contrib Nephrol 2010;166:73-82.

56 Bellomo R, Wan L, May C: Vasoactive drugs and acute kidney injury. Crit Care Med 2008; 36(4 suppl):S179-S186.

57 Landoni G, et al: Fenoldopam in cardiac surgery-associated acute kidney injury. Int J Artif Organs 2008;31:561.

58 Nigwekar SU, Waikar SS: Diuretics in acute kidney injury. Semin Nephrol 2011;31:523-534.

59 Chou YH, et al: Impact of timing of renal replacement therapy initiation on outcome of septic acute kidney injury. Crit Care 2011; 15:R134. 